\title{
Progress in Fabrication Technique of Carbon Nanotubes Reinforced Al Matrix Composites
}

\author{
WU Chun, MA WenXin, CHEN YaPing, LIYing CHEN Yan, LIJing
}

Bei'jing Hi-Tech Institute, Bei'jing,PRC, 100085,wuchun217@163.com

Keywords: Carbon nanotubes; Aluminum matrix composite; Reinforced phase

\begin{abstract}
The carbon nanotubes and carbon nanotubes reinforced aluminum matrix composite materials was described, the basic characteristics about carbon nanotubes preparation methods of aluminum matrix composites and preparation process problems and solutions. Finally, the carbon nanotubes reinforced aluminum matrix composites in the future research trends was analyzed.
\end{abstract}

\section{The introduction of carbon nanotubes}

Carbon nanotubes was founded in 1991 by Iijima Japan electron microscopy experts found in experiment [1] and the fifth way that the carbon allotropes (the first four, respectively is graphite, diamond, bucky balls and no qualitative carbon). Carbon nanotubes can be seen as a single layer or multi-layer graphite piece of curly and geometric seamless graphite tube. Each layer of nanotube consists of carbon atoms by sp2 hybrid structure with the surrounding three fully bonded carbon atoms of hexagonal plane of cylinder, pentagons or heptagon combination on both ends closed. So that the pipe produced in the cylinder body corresponding positive and negative bending curvature.

Carbon nanotubes because of its unique structure and performance in the nano composite material has good application prospect, theoretical calculation and the results show that carbon nanotubes have high elastic modulus, can reach $1 \mathrm{TPa}$ (modulus of elasticity of $1.2 \mathrm{TPa}$ diamond), even reached $1.8 \mathrm{TPa}$; Carbon nanotubes is about 100 times the steel intensity, compared with just about one 6 of the density. Carbon nanotubes have excellent plastic deformation, the elastic strain can reach $5 \%$, even up to $12 \%$, which is about 60 times of steel [2-6].

\section{Carbon nanotubes reinforced aluminum matrix composites}

Carbon nanotubes aluminum base composite material have low density, high strength, good oxidation resistance and corrosion resistance, etc, become a cutting-edge technology sector development research direction of high performance structural material, has become a hot research at home and abroad in recent years. Although carbon nanotubes aluminium matrix composites is still in trial stage, but has made great progress. Rice. University of Barrera, etc, fullerenes was used as aluminum enhanced phase pure aluminium hardness increased by nearly $80 \%$. Kuzumaki was prepared by hot pressing, hot extrusion process of carbon nano tube of aluminum base composite material, the elongation increased by about $40 \%$. And showed a good chemical stability. Ding Zhipeng the pressureless infiltration method is adopted in the molten metal into the precast block, the preparation of carbon nanotubes/aluminum composite material, found that when the volume fraction of carbon nanotubes is more than 15\%, the hardness of the composites with worse instead. Such as the use of hot extrusion of the CNTs/A1 composite material shows good electrical conductivity at low temperature. From the perspective of the properties of carbon nanotubes is excellent, at the moment, carbon nanotubes aluminum base composite material performance wasn't up to the theoretical value, the influence of carbon nanotubes elements of aluminum base composite material performance are also a lot, so the scientific workers needs further research.

\section{Preparation methods of carbon nanotube aluminium matrix composites}

\subsection{The powder metallurgy method}

Powder metallurgy method the preparation of metal matrix composites is the first process, the 
method is to mix CNTs with aluminum powder evenly stirring, and then to the ball grinding, drying, compaction and sintering, etc. The advantage of this method is mainly carbon nanotubes can be distributed evenly in the aluminum substrate, and can accurately control the content of carbon nanotubes, higher degree of freedom. CNTs/aluminum substrate is not easy to happen chemical reaction, technical process of equipment requirements is not high also. Defect is mixed powder longer pressure large and ball mill process of CNTs may be damage the original structure, the preparation of composite materials in general there are more pores, bubbles, cracks, etc., internal defects affecting the performance of the aluminum matrix composites, carbon nanotubes process is complex and the manufacturing cost is high, not easy preparation of complex shape parts.

\subsection{Stir casting process}

Stirring casting process is one of the most common method, the method is in carbon nanotubes were added to the molten liquid metal, when carbon nanotubes enhancement phase slowly added to the liquid metal, the mechanical agitator strong stirring, using vortex formed huge negative pressure forced the dispersed carbon nanotubes in metal matrix, so as to achieve the aim of dispersion, were dispersed uniformly carbon nanotubes reinforced metal matrix composites. The process is easy to operate, simple to equipment requirements is not high, high production efficiency, etc. Defect is due to the low carbon nanotube density relative metal, easy to rise in the melt metal, and lead to enhanced phase segregation, uneven dispersion and more porosity voids, the performance of the aluminum matrix composites of carbon nanotubes has very big effect.

\subsection{The melt impregnation method}

Melt impregnation is a metal melt penetrated under certain technological conditions has a certain shape and high gap of precast block, reach the role of infiltration, and then through the natural cooling, solidified to the preparation of composite materials. Generally melt impregnation method is divided into pressure infiltration and pressureless infiltration. Pressure infiltration effect is obvious, preparation of the composite material porosity is relatively low, there are reports that this approach to the preparation of $50 \%$ volume fraction of composite materials. But the complicated preparation process, equipment requirement is high, prone to biochemistry reaction, generate brittle material, impact on the performance of the composites. And typically only can be carried out under inert gas protection. Pressureless infiltration by definition in the natural state to a state of balance of melt impregnation.

\section{4 .The problems and solutions to problems}

CNTs excellent physical and chemical properties have made it an ideal aluminum matrix composite reinforced phase, therefore, in recent years, study of CNTs/A1 composite materials has become a hotspot of research on materials science, and carbon nanotubes reinforced aluminum matrix composites research has made some progress. But the experimental results and theoretical results have great difference, did not achieve ideal requirements, mainly in carbon nanotubes preparation of aluminum matrix composites has a lot of problems in the process, so the necessary solution is the top priority.

\subsection{Dispersion of carbon nanotubes in aluminum matrix problem}

Known as carbon nanotube density is small, high length-diameter ratio, high specific surface energy, the traditional liquid phase method and solid phase method is adopted when CNTs are partial or reunion, and preparation of the composite material properties is low. So, in order to improve the material performance, the key step is to improve the dispersion of carbon nanotubes, fascicles or assume the, avoid carbon nanotubes in aluminum substrate together to form weak phase. Because the weaker phase brittle interface, easy to cause fracture, especially when the carbon nanotubes in aluminum grain boundary point occurred when they were reunited, makes the grain boundary in intensity is reduced, which reduces the compound materials performance.

Current solution mainly include: (1) in situ synthesis: mainly chemical vapor deposition method is given priority to, in the metal substrate directly growing carbon nanotubes. The method in the preparation of carbon nanotubes dispersed carbon nanotubes at the same time, effectively and make carbon nanotubes and metal substrate combination, to reach the role of interface wettability. (2) 
mechanical stirring dispersion: mechanical mixing dispersion is one of the dispersion methods which are frequently used, mainly using the method of mechanical force strong stirring impact that nanoparticles fully dispersed in a medium of a form. (3) ultrasonic dispersion, using ultrasonic instantaneous energy released by the impact of carbon nanotubes so as to achieve the purpose of the dispersed particles. (4) dispersant dispersion: the dispersant dispersion mainly through adsorption dispersant to change particle surface charge distribution, introduction of special functional groups, creating static stability and stable space barrier function to achieve the dispersion effect. But, scattered in the use of dispersants, the dispersing agent dosage has certain limitation, excessive dispersant could damage the structure of carbon nanotubes. (5)Various methods of comprehensive use: for carbon nanotube dispersion technology at present, is usually a variety of dispersion method is used in combination to achieve the purpose of dispersion. As with strong acid oxidation and mechanical stirring dispersion. Comprehensive use various methods improved the dispersion efficiency on one hand, on the other hand, a certain extent, improved the dispersed degree.

\subsection{Carbon nanotubes and aluminum substrate wetting problem}

Effect of carbon nanotubes in the performance of the aluminum matrix composites play a factor in addition to the carbon nanotube dispersion problem and how to effectively solve the interface combination of carbon nanotubes and aluminum substrate, is the problem of wetting. We know because is nanomaterials, carbon nanotubes and high length to diameter ratio, so it is difficult to form solid carbon nanotubes and aluminum matrix interface, and the combination of carbon nanotubes, the outstanding performance is difficult to play out, and preparation of the composite material is difficult to achieve the ideal performance metrics. So must try to properly on the surface of the carbon nanotubes modified to improve the invasive of matrix. Solution to the problem at present mainly in the field of carbon nanotubes coated on the surface of special metal layer, generally for base metal. So that is invasive and not to introduce impurities.

There are researchers in carbon nanotubes, $\mathrm{Co}, \mathrm{Cu}, \mathrm{Ni}, \mathrm{Ag}$ plating the surface of the literature. Found after coating of carbon nanotubes on the one hand, combined with the matrix metal is good, the formation of more solid interface; On the other hand also promote the dispersion of carbon nanotubes in the substrate. But so far, the interface of carbon nanotubes reinforced aluminum matrix composites research literature is less, also can't get the system convenient solution. Therefore, further study of interface between carbon nanotubes and aluminum matrix based on sex, to reveal the carbon nanotubes reinforced aluminum matrix composites has guiding significance for strengthening mechanism.

\subsection{Strengthening mechanism}

Carbon nanotubes with nanoscale hollow pipe diameter, high length-diameter ratio, in general, under the natural state is curly linear distribution. Carbon nanotubes have a much bigger than a whisker of length to diameter ratio, but far less than the diameter size and fibre, therefore the enhancement mechanism of carbon nanotubes and whisker and fiber is different. In general carbon nanotubes reinforced metal matrix composites may enhance mechanism mainly include the mixed law, fine-grain strengthening mechanism, the dislocation strengthening and dispersion strengthening mechanism. Dispersion strengthening mechanism is the study of relatively mature enhancement mechanism, dispersion strengthening mechanism is mainly in the matrix composites, carbon nanotubes block matrix metal grew up and the dislocation motion, make the matrix metal plastic deformation is restrained, so as to improve material performance.

\section{Conclusion}

Due to the excellent physical chemical and mechanical properties of carbon nanotubes, with the composite metal materials after can get all kinds of high performance new composite materials, it has become the research of carbon nanotubes composites is a very important area. But how to effective use of the carbon nanotubes enhancement effect is a problem to be resolved in the future. At the moment, carbon nanotubes reinforced metal matrix composite materials research is welcome, but its method of preparation of oneness, the complexity of processing technology, production efficiency is not high, these problems also restricts the development of metal matrix composites, 
carbon nanotubes need further exploration research, only in this way can we make carbon nanotubes aluminum matrix composites have practical applications.

\section{Acknowledgements}

The authors would like to express appreciation to No.503 Faculty, Xi'an Research Institute of High Technology for their generous support to this work.

\section{References}

[1] Lijima S. Helical microtubIes of graphitic carbon[J]. Nature,1991,354:56-58.

[2] Gao G, Cagin T, Goddard W A. Energetics, structure, mechanical and vibration pro-perties of single-walled carbonnanotubes[J]. Nanotechnolgoy, 1998,9(3):184-191.

[3] Mishra R S, Ma Z Y. Frictionstir welding and processing[J]. Mater Sci Eng R: Reports,2005,50(1-2):1.

[4] Ghasemi-Nejhad, Mehrdad Ni, Askari Davood. Mechanical properties modeling of carbonsingwalled nanotubes [J]. A Finite Element Method. Journal of Computati-onal and Theoretical Nanoscience,2005,2(2):298-318.

[5] Fu Yiming, Xu Xiaoxian. Analysis of Material mechanical properties for single walled carbon nanotubes[J]. Acta. Mechan ma Solida Sinica, 2005,18(1):46-51.

[6] Tokunaga T, Kaneko K, et al. Production of aluminum-matrix carbon nanotube composite using high pressure torsion[J]. Mater Sci Eng A,2008,490(1-2):300. 